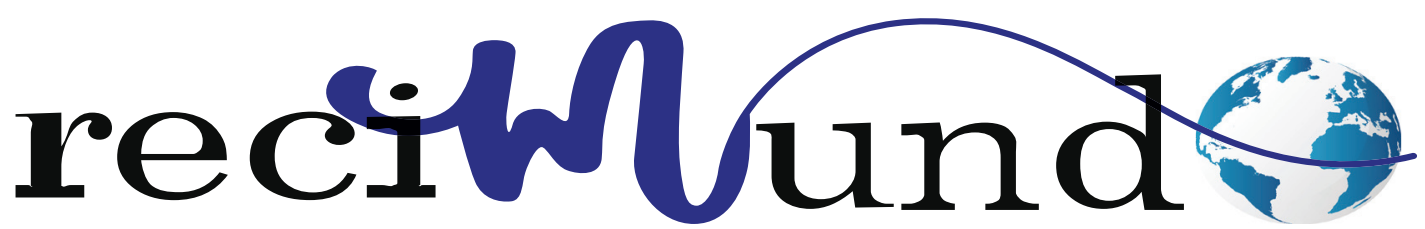

Revista Científica Mundo de la Investigación y el Conocimiento

DOI: 10.26820/recimundo/4.(4).octubre.2020.199-212

URL: http://recimundo.com/index.php/es/article/view/899

EDITORIAL: Saberes del Conocimiento

REVISTA: RECIMUNDO

ISSN: 2588-073X

TIPO DE INVESTIGACIÓN: Articulo de Investigación CÓDIGO UNESCO: 5801 Teoría y Métodos Educativos PAGINAS: $199-212$

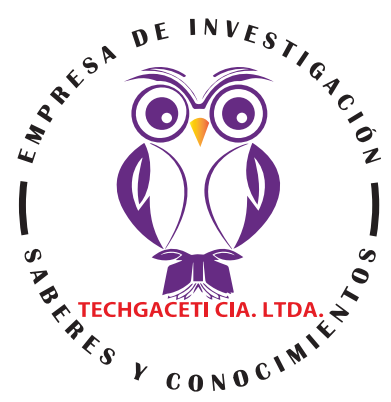

\title{
Las plataformas virtuales para fomentar aprendizaje colaborativo en los estudiantes del bachillerato
}

Virtual platforms to promote collaborative learning in high school students Plataformas virtuais para promover a aprendizagem colaborativa em alunos do ensino médio

Michael Antonio Tomalá De la Cruz'; Gabriel Giancarlo Gallo Macías;

José Luis Mosquera Viejó3;; Juan Carlos Chancusig Chisag ${ }^{4}$

RECIBIDO: 10/07/2020 ACEPTADO: 26/08/2020 PUBLICADO: 15/10/2020

1. Magister en Seguridad Informática Aplicada; Ingeniero en Sistemas; Universidad de Guayaquil; Guayaquil, Ecuador; mictomcruz@hotmail.com; iD https://orcid.org/0000-0003-1104-479X

2. Magister en Educación Superior Investigación e Innovaciones Pedagógicas; Diploma Superior en Pedagogía Universitaria; Ingeniero en Sistemas Computacionales; Universidad de Guayaquil; Guayaquil, Ecuador; gabriel.gallom@ug.edu.ec; (iD https://orcid.org/0000-0002-6924-544X

3. Magister en Gestión de la Productividad y la Calidad; Ingeniero Industrial; Universidad de Guayaquil; Guayaquil, Ecuador; josluis2002@hotmail.com; (iD https://orcid.org/0000-0001-9093-7451

4. Magister en Gestión de la Educación mención en Educación Superior; Especialista en Diseño Curricular; Diploma Superior en Gestión Prospectiva de la Educación; Ingeniero en Informática y Sistemas Computacionales; Universidad Técnica de Cotopaxi; Latacunga; juan.chancusig@utc.edu.ec; (D) https://orcid.org/0000-0002-5706-8845

CORRESPONDENCIA

Michael Antonio Tomalá De la Cruz

mictomcruz@hotmail.com

Guayaquil; Ecuador

(C RECIMUNDO; Editorial Saberes del Conocimiento, 2020 


\section{RESUMEN}

Las plataformas virtuales y su integración en el ámbito educativo permiten desarrollar competencias y destrezas, permiten trabajar en grupo de forma colaborativa, presentar ideas y respetar las de otros en un medio pluralista y de equidad social. Las plataformas educativas favorecen el proceso de formación virtual, esta investigación duró 5 meses y se desarrolló con una población estudiantil y profesores del segundo año de educación general unificado de la Unidad Educativa Ancón en la provincia de Santa Elena, que corresponde a 200 estudiantes y 40 docentes, respectivamente. El problema que se observó es el bajo nivel de aprendizaje en ciertas asignaturas de la especialidad especialmente en gestores de base de datos. Esta dificultad se deseaba resolver mediante trabajo colaborativo utilizando las herramientas tics a través de aula virtual. Se pudo identificar mediante una investigación de campo qué tipo de habilidades y destrezas son necesarias aplicar en los estudiantes para mejorar su aprendizaje en gestores de base de datos y como mejorar dichas habilidades y destrezas mediante trabajo colaborativo apoyado con tecnología. Estos resultados, permitieron desarrollar la propuesta que fue la implementación de un aula virtual de entrenamiento colaborativo para mejorar el rendimiento académico de los estudiantes. La tecnología seleccionada fue un aula virtual por cuanto demostró tener una gran cantidad de ventajas en relación con los otros. Una vez desarrollado, la plataforma virtual fue probada y validada con un grupo de profesores y estudiantes, teniendo como resultado una gran aceptación y buenas perspectivas en su uso.

Palabras clave: Aprendizaje colaborativo, plataformas virtuales, Aula virtual, educación virtual.

\section{ABSTRACT}

Virtual platforms and their integration in the educational field allow the development of competencies and skills, allow collaborative group work, present ideas and respect those of others in a pluralistic and socially equitable environment. The educational platforms favor the virtual training process, this research lasted 5 months and was developed with a student population and teachers of the second year of unified general education of the Ancón Educational Unit in the province of Santa Elena, which corresponds to 200 students and 40 teachers, respectively. The problem that is carried out is the low level of learning in certain specialty subjects, especially in database managers. This difficulty is to be solved through collaborative work using the ICT tools through the virtual classroom. It was possible to identify through a field investigation what kind of skills and abilities are necessary to apply in students to improve their learning in database managers and how to improve these skills and abilities through collaborative work supported with technology. These results allowed to develop the proposal that was the implementation of a virtual classroom for collaborative training to improve the academic performance of students. The technology selected was a virtual classroom in that it proved to have a great number of advantages in relation to the others. Once developed, the virtual platform was tested and validated with a group of teachers and students, resulting in great acceptance and good prospects for its use.

Keywords: Collaborative learning, virtual platforms, virtual classroom, virtual education.

\section{RESUMO}

As plataformas virtuais e sua integração no campo educacional permitem o desenvolvimento de competências e habilidades, permitem o trabalho colaborativo em grupo, apresentam ideias e respeitam as dos outros em um ambiente pluralista e socialmente justo. As plataformas educacionais favorecem o processo de formação virtual, esta pesquisa teve duração de 5 meses e foi desenvolvida com uma população de alunos e professores do segundo ano do ensino geral unificado da Unidade Educacional Ancón na província de Santa Elena, que corresponde a 200 alunos e 40 professores, respectivamente. O problema que se coloca é o baixo nível de aprendizado em certas disciplinas da especialidade, especialmente em gerentes de banco de dados. Essa dificuldade deve ser contornada por meio do trabalho colaborativo utilizando as ferramentas TIC por meio da sala de aula virtual. Foi possível identificar por meio de uma investigação de campo que tipo de habilidades e habilidades são necessárias para aplicar nos alunos para melhorar seu aprendizado em gerenciadores de banco de dados e como melhorar essas habilidades e habilidades por meio de um trabalho colaborativo apoiado em tecnologia. Esses resultados permitiram desenvolver a proposta que consistia na implantação de uma sala de aula virtual para treinamento colaborativo para melhorar o desempenho acadêmico dos alunos. A tecnologia escolhida foi a sala de aula virtual, pois demonstrou ter um grande número de vantagens em relação às demais. Depois de desenvolvida, a plataforma virtual foi testada e validada com um grupo de professores e alunos, resultando em ótima aceitação e boas perspectivas de uso.

Palavras-chave: Aprendizagem colaborativa, plataformas virtuais, sala de aula virtual, educação virtual. 


\section{LAS PLATAFORMAS VIRTUALES PARA FOMENTAR APRENDIZAJE COLABORATIVO EN LOS ESTUDIAN- TES DEL BACHILLERATO}

\section{INTRODUCCIÓN}

Al hablar de aprendizaje colaborativo se hace referencia al desarrollo de habilidades y competencias que son necesarias en los estudiantes, pero que a su vez permitan el surgimiento de otras experiencias y dinámicas de trabajo dentro del aula.

En la época actual las Tecnologías de la Información y la Comunicación están surgiendo nuevas formas de planificar y desarrollar los procesos de Enseñanza-Aprendizaje, de manera que se promueva la participación sincrónica y asincrónica entre los sujetos que no se encuentran físicamente en el mismo lugar, ni en las mismas coordenadas temporales, se presentan cada vez más de una manera natural y al alcance de todos; por lo tanto, no son ajenas a la educación, sobre todo cuando desde su inicio se han adaptado para ser usadas como apoyo a las prácticas tradicionales de enseñanza

Según Fernández y Valverde ( 2013), “Los entornos virtuales de aprendizaje ofrecen una serie de posibilidades para procesos de colaboración, donde el alumnado produce conocimiento de forma activa, formulando ideas que son compartidas y construidas a partir de las reacciones y respuestas de los demás"

Entonces en nuestro labor docente se deben emplear metodologías y herramientas tecnológicas que nos permitan seguir trabajando en la construcción del conocimiento, al aspecto social, la interacción y al pensamiento crítico que les permita buscar la información, analizarla, compartirla, editar nueva a partir de ésta y especialmente, aprender a gestionar sus propios procesos de aprendizaje así como diseñar materiales que faciliten la adquisición de habilidades y competencias en cada grupo de estudiantes y como docentes debemos facilitarles ese camino.

Según Álvarez, et.al,. (2005) Afirma:
"Que el aprendizaje colaborativo se caracteriza por no contemplar al aprendiz como persona aislada, sino en interacción con los demás, pues compartir objetivos y distribuir responsabilidades son formas deseables de aprendizaje, y por potenciar el rol de las herramientas tecnológicas como elementos mediadores en este proceso, facilitando los procesos de interacción y la solución conjunta de los problemas".( p.4).

Se debe tener en cuenta que no basta con poner a un grupo a interactuar para que se produzca el aprendizaje, sino que se deben articular los diferentes elementos para Ilegar a procesos conjuntos de intercambio y construcción del conocimiento.

\subsection{ANTECEDENTE}

Gran parte de las investigaciones de aprendizaje colaborativo y plataformas virtuales en el ámbito nacional como en lo internacional están orientadas en las variables relacionadas a la construcción de conocimiento, al aspecto social, la interacción, el pensamiento crítico, y las plataformas virtuales como estrategia didáctica, como aportación para mejorar el aprendizaje en los estudiantes.

El contenido de este articulo está relacionados con el problema de tal forma que puedan ser manejados en acciones concretas, tiene el propósito de dar a la investigación un sistema coordinado y coherente de conceptos y proposiciones que nos permitirán explicar mejor la investigación que realizamos, de tal modo que permitan orientar nuestra búsqueda y nos ofrece una conceptualización adecuada de los términos que utilizamos

\subsection{APRENDIZAJE COLABORATIVO}

El aprendizaje colaborativo se sustenta en teorías cognoscitivas. Para Piaget hay cuatro factores que inciden e intervienen en la modificación de estructuras cognoscitivas:

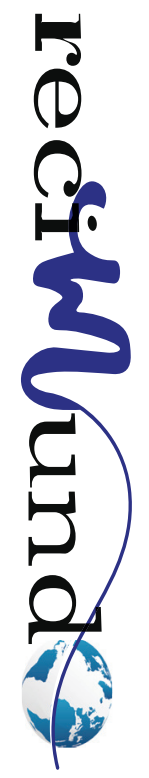


la maduración, la experiencia, el equilibrio y la transmisión social. Todos ellos se pueden propiciar a través de ambientes colaborativos. En la teoría constructivista (Vigotsky, 1974), el aprendiz requiere la acción de un agente mediador para acceder a la zona de desarrollo próximo, éste será responsable de ir tendiendo un andamiaje que proporcione seguridad y permita que aquél se apropie del conocimiento y lo transfiera a su propio entorno.

También se podría definir al aprendizaje colaborativo como aquellas metodologías de aprendizaje que incentivan la colaboración entre individuos para conocer, compartir, y ampliar la información que cada uno tiene sobre un tema. Estos pueden ser o no apoyados con tecnología, así como estrategias para propiciar el desarrollo de habilidades mixtas (aprendizaje y desarrollo personal y social) donde cada miembro del grupo es responsable tanto de su aprendizaje como del de los restantes del grupo.

\subsection{APRENDIZAJE COLABORATIVO $Y$ CONSTRUCTIVISMO}

El aprendizaje colaborativo se sustenta en teorías cognoscitivas de Piaget hay cuatro factores que inciden e intervienen en la modificación de estructuras cognoscitivas: la maduración, la experiencia, el equilibrio y la transmisión social. Todos ellos se pueden propiciar a través de ambientes colaborativos. En la teoría constructivista Vigotsky (1974), el aprendiz requiere la acción de un agente mediador para acceder a la zona de desarrollo próximo, éste será responsable de ir tendiendo un andamiaje que proporcione seguridad y permita que aquél se apropie del conocimiento y lo transfiera a su propio entorno. En cuanto a las implicaciones educativas de los anterior.

Según Coll y Solé (1990) definen a la enseñanza como un proceso continuo de negociación de significados, de establecimiento de contextos mentales compartidos, fruto y plataforma, a su vez, del proceso de negociación, lo que permite verificar las conexiones entre aprendizaje, interacción y cooperación: los individuos que intervienen en un proceso de aprendizaje, se afectan mutuamente, intercambian proyectos y expectativas y replantean un proyecto mutuo, que los conduzca al logro mutuo de un nuevo nivel de conocimiento y satisfacción (p.332)

\subsection{HERRAMIENTAS COLABORATIVAS VIRTUALES MODERNAS PARA FOMEN- TAR EL APRENDIZAJE COLABORATIVO EN EL AULA}

Las herramientas colaborativas modernas por excelencia y las más usadas en la actualidad son: Wikipaces, Moodle, Edmodo, todos ellos son excelentes opciones para diversos propósitos educativo, aunque existen muchos más.

\subsection{Moodle}

Según la página oficial, docs.moodle.org/ "Moodle es una plataforma de aprendizaje diseñada para proporcionarle a educadores, administradores y estudiantes un sistema integrado único, robusto y seguro para crear ambientes de aprendizaje personalizados".

La plataforma Moodle permite al docente crear su propio entorno virtual de aprendizaje sin tener mucho conocimiento primero porque es fácil de usar y sobre todo es gratuito donde el docente y el estudiante interactúan entre si con actividades en línea, el docente organiza y diseña curso para que el estudiante se suscriba y realice dicho curso de forma simultánea.

Autores como (Ortiz \& Hernández, 2013), consideran que Moodle es un sistema de gestión de cursos, que es distribuido libremente, y que es una ayuda muy buena que docentes y educadores en general puedan crear comunidades de aprendizaje en línea, a este tipo de herramienta tecnológica se la 


\section{LAS PLATAFORMAS VIRTUALES PARA FOMENTAR APRENDIZAJE COLABORATIVO EN LOS ESTUDIAN- TES DEL BACHILLERATO}

conoce como LMS (Learning Management System).

\subsection{Edmodo}

Según la página oficial https://www.edmodo.com/; Edmodo es "Una plataforma social educativa gratuita que permite la comunicación entre los alumnos y los profesores en un entorno cerrado y privado a modo de microblogging. Fue fundada en el 2008 por Jeff O'Hara y Nic Borg. Recientemente fue adquirida por Revolution Learning (Learn Capital). El número de usuarios se ha ido incrementando notablemente con más de más de 43 millones de profesores y estudiantes que ya se conectan en aulas en línea, colaborando en asignaciones, descubriendo recursos nuevos y más".

- El programa es un espacio de apoyo para la construcción de conocimiento que se genera a partir de las contribuciones individuales y grupales de los participantes.

- Permite categorizar las aportaciones realizadas durante el proceso de aprendizaje con objeto de facilitar la reflexión sobre el contenido de la propia intervención.

- Permite crear un espacio virtual de comunicación con tu alumnado, profesores incluso padres de familia, en el que se puede compartir mensajes, archivos y enlaces, un calendario de trabajo, así como proponer tareas, exámenes, cuestionarios, y actividades entre otras.

\subsection{PLATAFORMAS VIRTUALES}

Según la plataforma http://www.interclase. com/ Las plataformas virtuales facilitan a profesores la gestión de cursos virtuales para sus estudiantes, especialmente ayudándolos en la administración y desarrollo del curso virtuales para estudiantes, las cuales reciben distintos nombres, tales como "entornos virtuales de enseñanza y aprendizaje" o "entornos de aprendizajes integrados", "ambiente virtual de aprendizaje", "Sistemas de Gestión del Aprendizaje", "Sistema de Gestión de Curso", "Sistema de Gestión de Contenido para el Aprendizaje", "Ambientes de Aprendizaje Gestionado", "Sistema de Apoyo al Aprendizaje", "Plataforma de Aprendizaje" .

Sebastián Díaz (2009) las define como "un entorno informático en el que nos encontramos con muchas herramientas agrupadas y optimizadas para fines docentes. Su función es permitir la creación y gestión de cursos completos para internet sin que sean necesarios conocimientos profundos de programación".

\subsection{AULA VIRTUAL}

Dadas que el curso está basado en la educación a distancia apoyada en tecnologías de la información, es necesario el empleo de una Plataforma para la Administración del Aprendizaje. (Learning Management Sistem, LMS) comúnmente denominada Aula Virtual.

El Aula Virtual es una herramienta que brinda las posibilidades de realizar enseñanza en línea. Es un entorno privado que permite administrar procesos educativos basados en un sistema de comunicación mediado por computadoras. De manera que se entiende como Aula Virtual, al espacio simbólico en el que se produce la relación entre los participantes en un proceso de enseñanza y aprendizaje que, para interactuar entre sí y acceder a la información relevante, utilizan prioritariamente un sistema de comunicación mediada por computadoras.

Las plataformas virtuales, son programas (software) orientados a la Internet, se utilizan para el diseño y desarrollo de cursos o módulos didácticos en la red internacional. Permiten mejorar la comunicación (alumno-docente; alumno-alumno) y desarrollar el aprendizaje individual y colectivo. 


\subsection{LA EDUCACIÓN VIRTUAL}

Dentro del contexto de transformación cultural se concibe como un sistema abierto y permanente fundamentado en un nuevo enfoque pedagógico que favorece el estudio autónomo e independiente del estudiante, la tecno-logía informático-telemática y sus contribuciones al campo del aprendizaje ayudan a la creación de un nuevo paradigma de interacción socioeducativo virtual. Justamente la educación virtual, como ya se ha expresado en otras ocasiones.

Vera y Morales (2005). "El acto educativo adquiere nuevos matices a partir de la inserción del software educativo y de la consideración como objeto de los procesos de enseñanza y aprendizaje." (pág. 52) En la actualidad la aplicación de programas informáticos hace que el aprendizaje de una determinada asignatura conlleve a obtener un mejor rendimiento académico y por consiguiente que se fijen mejor los contenidos

\subsection{Recursos tecnológicos que fomen- tan aprendizaje colaborativo}

\subsubsection{Correo electrónico}

El correo electrónico es una herramienta que permite el intercambio de información y documentación entre diferentes personas. Por lo general se utiliza para el envío de texto, sin embargo, también admite el envío de archivos multimedia (imagen, texto, sonido y video).

Esta plataformas educativas priorizan el objetivo en la institución por lo que son muy económico y presta un servicio en línea y se puede aplicar trabajo colaborativo, uso de la nube, el programa Word de forma remota es igual que usarlo de forma local de escritorio de tal manera que los estudiantes forman, trabajan con el mismo tema asignado por el profesor desarrollando desarrollan sus propias conclusiones y terminan el trabajo antes del tiempo estipulado.
El correo electrónico tiene una gran ventaja porque facilita una conversación más abierta y democrática entre profesores y los estudiantes porque se sienten algunas veces más cómodos a formular preguntas y dudas a los profesores que en una situación de prespecialidad, de forma que los profesores son percibidos como más accesible. Su utilización también les favorece que trabajen más fuera del entorno de clase.

\subsubsection{Foros}

"El uso de foros de discusión en línea como una herramienta para el aprendizaje colaborativo requiere que los estudiantes se perciban mutuamente como personas reales y generen lazos afectivos entre ellos" (Santos A, 2011, p.1).

Este instrumento de comunicación es una herramienta asincrónica en la cual se desarrollan debates o discusiones sobre un tema en particular, a partir de una temática inicial los participantes realizan sus intervenciones, las cuales pueden ser leídas por todos los integrantes del curso, los foros lo podemos ver casi en todas las plataformas educativas de aprendizajes son Es muy útil para encontrar soluciones a problemas, y porque permiten que varias personas den su opinión en tiempo real sobre un tema especial, ayudando a dar respuesta a la pregunta inicialmente planteada

\subsubsection{Chat}

Tal como su nombre lo indica significa charla, y se basa en la comunicación sincrónica que establecen un grupo de personas, en línea y en tiempo real, puede ser sólo texto o texto y audio. Esta herramienta se da entre dos o más personas y es utilizada por lo general para compartir la discusión o el análisis sobre tema o recibir orientaciones en grupo por parte de un Tutor o Especialista.

\subsection{OBJETIVO GENERAL}




\section{LAS PLATAFORMAS VIRTUALES PARA FOMENTAR APRENDIZAJE COLABORATIVO EN LOS ESTUDIAN- TES DEL BACHILLERATO}

Evaluar la incidencia del uso de las plataformas virtuales como herramienta en el aprendizaje colaborativo de los estudiantes del segundo Año de Bachillerato en la Unidad Educativa Ancón en la Provincia de Santa Elena

\section{MATERIALES Y MÉTODOS}

El método de investigación es un trabajo de campo, para la búsqueda de la información que se usó en este trabajo una revisión exploratoria directa, como documentos de prensa, artículos científicos, documentales de artículos de periódicos digitales y prensa, revistas científica como Scielo los temas fueron aprendizaje colaborativo, educación, plataformas virtuales, La exploración se la efectuó en Metabuscadores como Google y Google Académico, Ask, Bing, libros y trabajo finales de tesis en las bases de las universidades importantes del Ecuador. Las palabras más usadas para la búsqueda fueron: aprendizaje colaborativo, trabajo colaborativo AND plataformas virtuales.

Para lograr los objetivos de la investigación se diseñó una encuesta realizada a 200 estudiantes entre edad de 16-18 años y 40 docente de dos instituciones que cuenta con la especialidad de Informática UEAncón,
UE Península de Santa Elena de la provincia de Santa Elena, mediante un muestreo probabilístico, la muestra es finita por eso se utilizó el muestreo aleatorio simple según Mario (2013).

Las entrevistas se efectuaron a las autoridades de las instituciones educativas de 5 colegios de la provincia de Santa Elena, en donde fueron escogidas: Guillermo Ordoñez, Santa Elena, Península de Santa Elena, Luis Celleri Avilés, Muey; con la finalidad de establecer criterios respecto a las estrategias que se utilizaron para mejorar el aprendizaje en ciertas asignatura base de la especialidad de Administración de Sistemas.

\subsection{PROCESAMIENTO Y ANÁLISIS}

La información se procesó en función de las técnicas utilizada en la investigación. En lo que se refiere a la tabulación, se utilizó el programa estadístico SPSS 10.1.

\section{RESULTADOS}

CONOCIMIENTO DE LOS DOCENTES SOBRE EL MANEJO DE LAS HERRAMIENTAS TICS QUE PROPICIAN APRENDIZAJE COLABORATIVO.

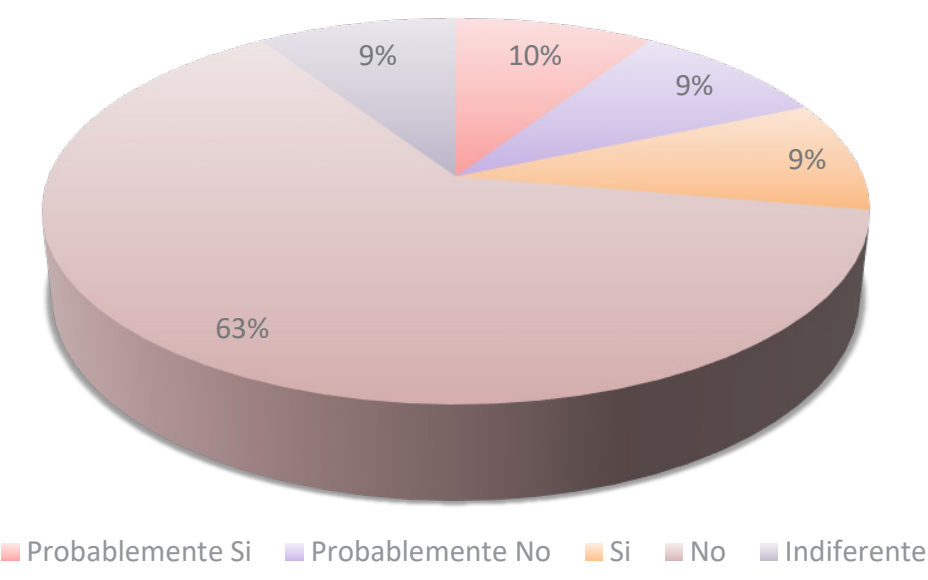

Gráfico 1. ¿Has recibido capacitación de tics uno y tics dos en los últimos cuatro años del Sí profe del Ministerio de Educación?

Fuente: Los autores 
Es necesario determinar el nivel de conocimiento de la población de estudio, acerca de del uso de las plataformas virtuales que se aplicada en el aula en las unidades educativas de la provincia de Santa Elena, de esta manera, se podrá establecer las estrategias orientada a la implementación de los tics como son las diversas plataformas educativas que en la actualidad hay muchas y funcionan de manera gratuitas solo es necesario suscribirse y diseñar los curso. Se determinó que la mayor parte de los docentes encuestados no tienen conocimiento, debido que las gestiones que realizan las autoridades de cada plantel no contribuyen a reforzar el conocimiento sobre estrategias metodología para aplicarlas en clase.

En la actualidad es necesario emplear herramientas tecnológicas idóneas que propicien trabajo colaborativo y que se enfoquen a la malla curricular del nuevo currículo educativo en base a las asignaturas de la especialidad y del perfil del estudiante, con el fin de ser altamente competitivos, y buscar nuevas estrategias con el uso de las plataformas educativa con el fin de obtener una educación de calidad y calidez.

\subsection{El uso de plataformas virtuales y recurso tecnológicos asincrónico y sincrónico como apoyo a la educación en los colegios fiscales de la provincia de santa Elena}

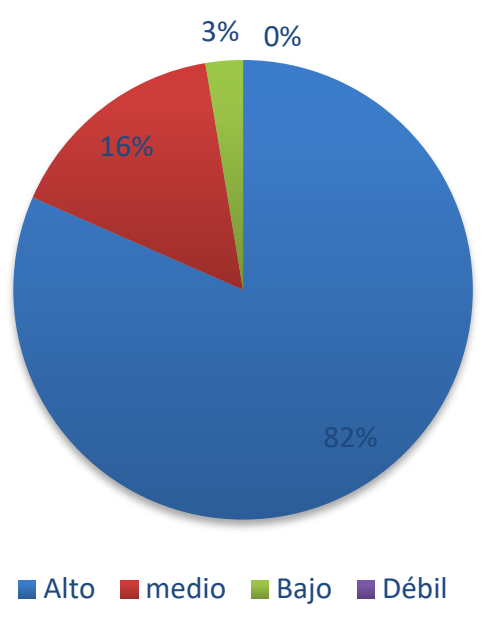

Gráfico 2. El uso de plataformas virtuales y recurso tecnológicos asincrónico y sincrónico como apoyo a la educación en los colegios fiscales de Santa Elena.

Fuente: Los autores

La mayor parte de encuestados de las instituciones educativas involucradas en la provincia de Santa Elena identifican que las plataformas educativas garantizan un mejor aprendizaje, que hay mayor participación en clase y más flexible al servicio de los estudiantes, mientras que un mínimo porcentaje las identifican como un gestor de aprendizaje de calidad educativa que buscando asegurar la continuidad de su formación durante toda su vida profesional. 


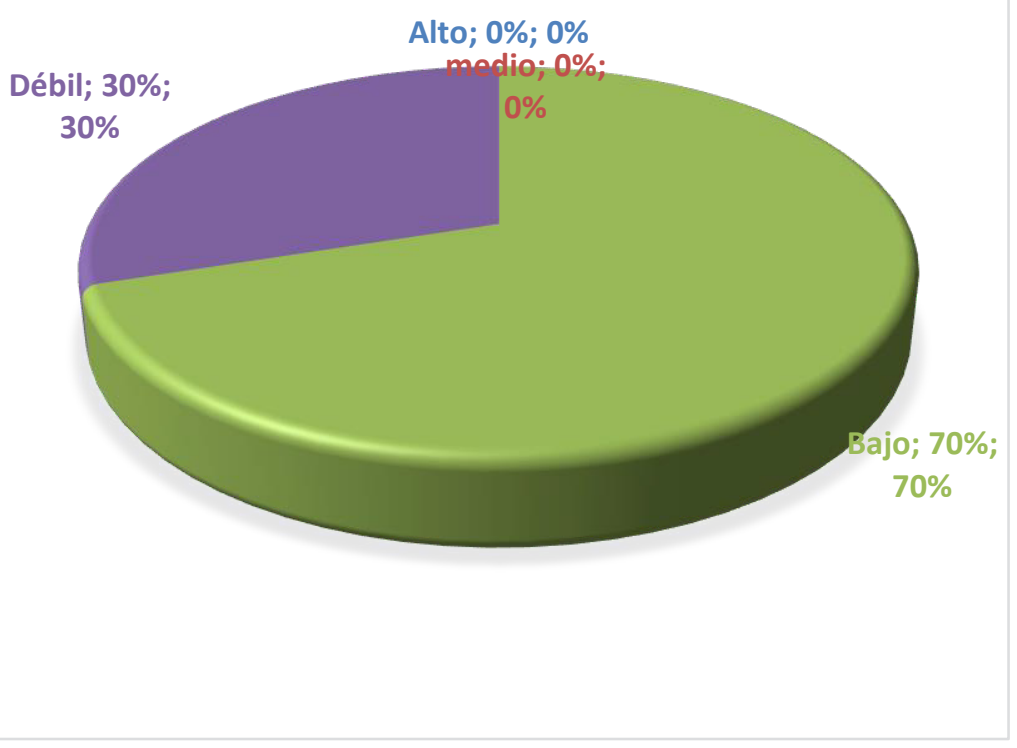

Gráfico 3. Implementación de una plataforma virtual como apoyo al aprendizaje de la provincia de Santa Elena

\section{Fuente: Los autores}

La Implementación de un entorno educativo en los colegios fiscales de la Provincia de Santa Elena es muy baja, muchas veces no se implementa por desconocimiento o miedo a los cambios tecnológicos o simplemente por no querer invertir en algo diferente como es la tecnología que bien le hace a la sociedad educativa.

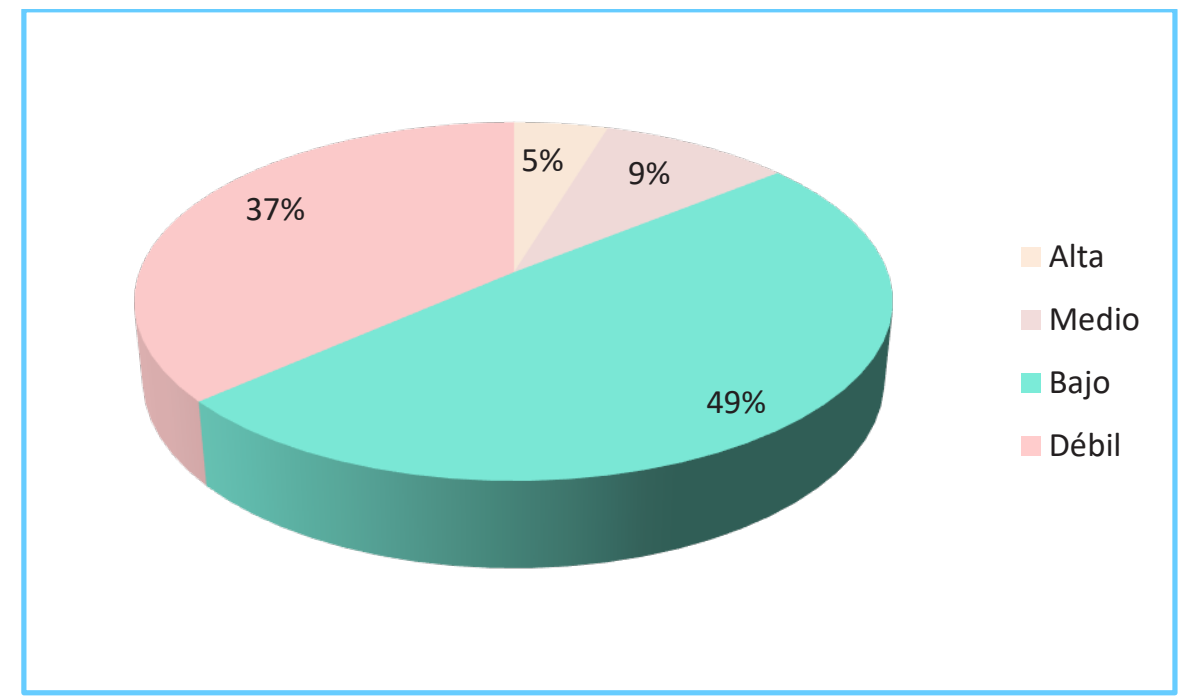

Gráfico 4. Nivel de posicionamiento de las plataformas educativas como apoyo al aprendizaje en la provincia de Santa Elena

Fuente: Los autores 


\subsubsection{Análisis del uso de las plataformas virtuales para impartir clases a través de in- ternet sin necesidad de tener conocimientos de programación.}

\begin{tabular}{|l|c|c|c|c|c|}
\hline \multicolumn{1}{|c|}{\begin{tabular}{c} 
Conceptos \\
\cline { 2 - 5 }
\end{tabular}} & $\begin{array}{c}\text { Muy } \\
\text { Elevado, o } \\
\text { Alto }\end{array}$ & $\begin{array}{c}\text { Elevado, o } \\
\text { Sobre lo } \\
\text { Normal }\end{array}$ & $\begin{array}{l}\text { Medio } \\
\text { Normal }\end{array}$ & $\begin{array}{c}\text { Bajo, o } \\
\text { Bajo lo } \\
\text { Normal }\end{array}$ & $\begin{array}{c}\text { Muy bajo, } \\
\text { o Pobre }\end{array}$ \\
\hline $\begin{array}{l}\text { Recomiendan la implementación de Centro de } \\
\begin{array}{l}\text { Formación Permanente e-learning a } \\
\text { instituciones educativa }\end{array}\end{array}$ & & & & \\
\hline $\begin{array}{l}\text { Se educa permanentemente en plataformas } \\
\text { educativas a los estudiantes del bachillerato. }\end{array}$ & & & & & \\
\hline $\begin{array}{l}\text { Hablan favorablemente acerca de las } \\
\text { plataformas educativas }\end{array}$ & & & & \\
\hline $\begin{array}{l}\text { Diseñan o implementan servicios que ofrecen } \\
\text { educación Online }\end{array}$ & & & & & \\
\hline
\end{tabular}

Matriz 1. Análisis del uso de las plataformas virtuales para impartir clases a través de internet sin necesidad de tener conocimientos de programación

Fuente: Los autores

\begin{tabular}{|l|l|l|}
\hline $\begin{array}{l}\text { Herramientas virtuales para el } \\
\text { apoyo de las clases presenciales }\end{array}$ & Calificación & \multicolumn{1}{|c|}{ ¿Por qué? } \\
\hline Edmodo & 5 & $\begin{array}{l}\text { Es una plataforma tecnóógica, educativa, gratuita } \\
\text { que permite la comunicación entre los alumnos, } \\
\text { profesores y padres de familia en un entorno } \\
\text { cerrado y privado muy fácil de usar y reparte } \\
\text { recurso en todo el mundo para mejorar sus } \\
\text { aprendizajes }\end{array}$ \\
\hline Moodle Unidad Educativa Ancón & & \\
\hline Edu 2.0 & & \\
\hline Diipo & & \\
\hline Midess Classroom & & \\
\hline
\end{tabular}

Matriz 2. Uso de varias plataformas virtuales que fomentan aprendizaje colaborativo de instituciones educativa en provincia de Santa Elena

Fuente: Los autores

Por tratarse de una herramienta virtual para el apoyo en el aprendizaje Edmodo es la plataforma más usada por millones de docentes a nivel mundial y en las instituciones educativas de la provincia de santa Elena por ser una red social que no exige instalación ni configuración local en el equipo ya que todo está basado en una aplicación en la red, además es una de las 32 plataformas educativas en línea más innovadora. 
3.3. Resumen de las plataformas virtuales educativas que fomenten aprendizaje colaborativo en el aula en las instituciones educativas de la provincia de Santa Elena

\begin{tabular}{|l|c|c|c|c|c|}
\hline CONCEPTO & 5 & 4 & 3 & 2 & 1 \\
\hline Que generaren prácticas colaborativas & & $\mathrm{X}$ & & & \\
\hline Resolver problemas, tareas grupales o autónomas & & & $\mathrm{X}$ & & \\
\hline Trabajo en equipo usando herramientas colaborativas & & & $\mathrm{X}$ & & \\
\hline Creatividad e imaginación & & & $\mathrm{X}$ & & \\
\hline Habilidades de comunicación & & & $\mathrm{X}$ & & \\
\hline Habilidades de aprendizaje & & & \\
\hline
\end{tabular}

Matriz 3. Resumen de temas que requieren atención

\section{Fuente: Los autores}

Las instituciones educativas de la provincia de Santa Elena necesitan crear su propia plataforma educativa porque cuenta con profesionales en el medio e instituciones especializadas en diseños web como la Universidad UPSE y la Unidad Educativa Ancón quienes tienen la especialidad de Informática, con ellas se puede realizar convenio para que para que se implementen las plataforma educativas para que genere beneficios y sobre todo propicien aprendizaje colaborativo a largo plazo, a través de la creación de recursos en la plataforma educativa con un valor agregado para lograr explotar esta ventaja educativas y esto se lo logra con una cultura de servicio dirigida al estudiante y al padre de familia, que garantice la ejecución de acciones de servicio y no existan barreras en relación a diferente cultura dentro del servicios prestados por las instituciones educativas.

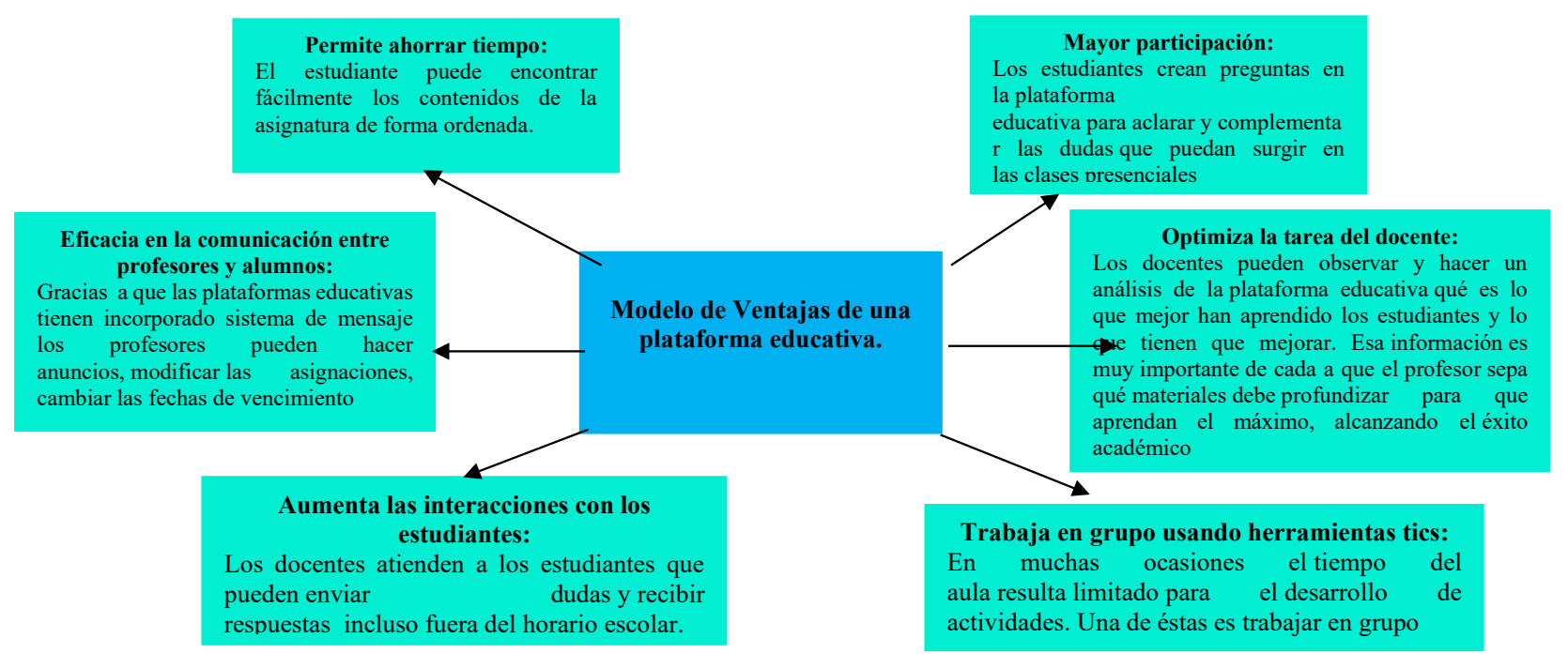

Ilustración 1. Modelo de ventaja de una plataforma educativa.

Fuente: Los autores 


\section{DISCUSIón}

\section{1 pasos para definir la incorporación de una plataforma educativa en la educación del bachillerato}

Según (Buzon García, 2008), Para definir la incorporación de una plataforma educativa como apoyo al aprendizaje colaborativo se debe la teleformación que se caracteriza sobre todo interacción y la comunicación entre el profesor y el alumnos y los contenidos de aprendizaje", además se debe considerar, como marco de referencia, se pueden seguir lo siguiente pasos: Analizar el costo del servicio online, identificar las diferencias del software si es de pago o código abierto, visualizar las principales características de las plataforma en cuanto a pagos y objetivos a cumplir, y alinear las características del servicio a los requerimientos del estudiante.

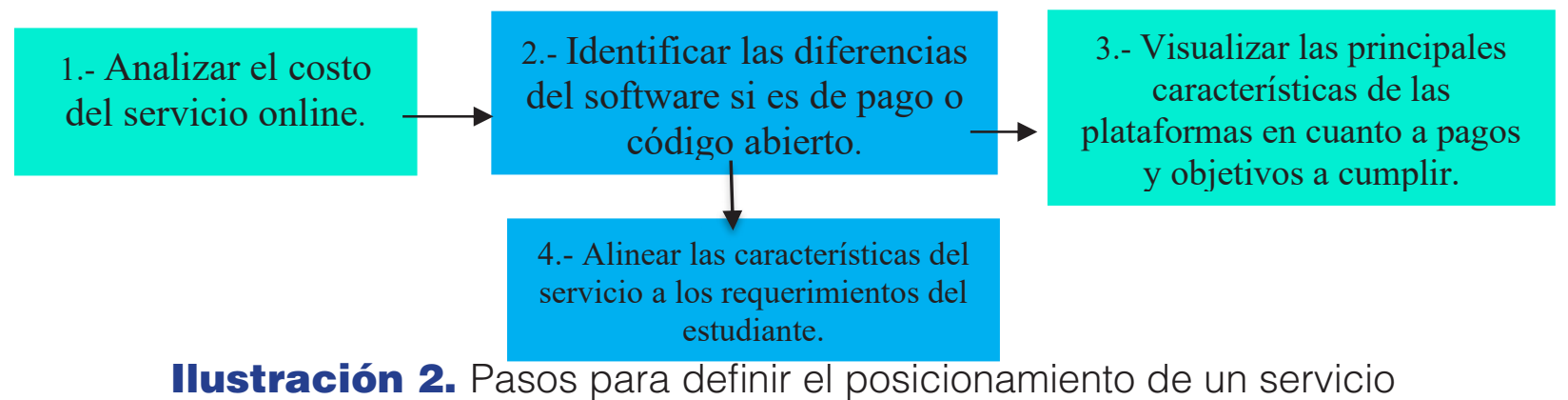

Fuente: Los autores

\subsection{EDUCACION DE CALIDAD}

(Olmos Gómez \& Tomé Fernández, 2017) "Se debe entender la calidad como un concepto abstracto tan amplio en definición y aplicación que cada organización debe entenderlo desde sus propios intereses $\mathrm{Y}$ por tanto, el concepto de calidad es el resultado de un acuerdo entre quienes pretenden establecer rasgos sobre determinado objeto o asunto en un espacio, tiempo y condiciones determinados".

(Sverdlick \& Ingrid, 2012), "Señalan que la calidad en relación con la gestión en educación fue extrapolada desde el ámbito empresarial y fue vinculada a la idea del control en el proceso de producción. Cuando el concepto de calidad se aplica al ámbito educativo es necesario considerar la posición desde la que se lo hace, ello implica finalidad, sentido y funciones de la educación"

\section{CONCLUSIONES}

- Debido a la limitada gestión económica para la incorporación de estrategia de aprendizaje y satisfacción de los estudiantes por parte de los colegios de la provincia de Santa Elena, se origina la débil enseñanza y poco uso de las herramientas tics aliada a la educación, debido a que no se emplean de forma idónea acciones estratégicas orientas a consolidar a una educación de calidad en las unidades educativas, y por ende la participación de los estudiantes es nula, así como el fortalecimiento de las plataformas educativas.

- En relación a la inexistencia de capacitaciones al personal docente de los colegios de la provincia de Santa Elena, se evidencia desconocimiento de los tipos de software que se deben usar en las instituciones educativas y por ende se 
genera una débil conformidad del padre de familia, por ser inadecuado el ambiente de trabajo generando así la insatisfacción de los educando, es necesario que se fortalezca esta carencia, para aportar a la excelencia educativa en los estudiantes de la comunidad peninsular.

- Debido a la limitada incorporación de estrategias didáctica que fomente la actividad colaborativa en los estudiantes, no se mejora de forma positiva el aprendizaje, en relación a las percepciones y preferencias de los padres, por ello no existen sólidas relaciones individuales entre los estudiantes y las docentes de la unidades educativas de la provincia de Santa Elena, originado por la inexistencia de un proceso personalizado de las herramientas colaborativas que permita conservar interés a los jóvenes en mejorar la calidad educativa.

- En relación, al desconocimiento de necesidades de los estudiantes por parte de las unidades educativa, no se han enfocado la atención al educando de forma idónea, ni los medios de comunicación, por ende es débil la relación entre padre de familia, estudiante y autoridades de los colegios, debido a los inadecuados vínculos de interacción y comunicación.

\section{Bibliografía}

Sverdlick, \& Ingrid. (2012). BUSCANDO A LA CALIDAD EDUCATIVA. Consejo Latinoamericano de Ciencias Sociales (CLACSO).

Albert Gomez, M. J. (2007). La Investigación Educativa: Claves Teoricas (Primera Edicion ed.). (J. M. Cejudo, Ed.) Madrid, España: McGraw Hill.

Bernal, C. A. (2010). Metodologia de la Investigación (Tercera Edición ed.). (O. Fernández Palma, Ed.) Bogotá, Colombia: Pearson Education.

BERNAL, C. (2010). Métodología de la Investigación. (Tercera Edición ed.). Bógota, Colombia: Pearson Educación.

Buzon García, H. (2008). La incorpoarcion de las plataforma virtuales a la enseñanza. Revista Latinoamerica de Tecnologia Educativa, 24.
Celaya, J. ( 2009). La empresa en la Web 2.0. El impacto de las redes sociales y las nuevas formas de comunicación online en la estrategia empresarial. . España: Gestión 2000.

ECHEVERRI CAÑAS, L. M. (2009). MARKETING PRÁCTICO. MADRID, ESPAÑA: STARBOOK.

Fernández, M. R., \& Valverde, J. (2013). Comunidades de práctica: un modelo de.

Global, T. (2005). wikispaces. Obtenido de https:// www.wikispaces.com/content/classroom

Inec. (2010). Inec Santa Elena. Santa Elena.

Lerma González, H. D. (2009). Propuesta, anteproyecto y proyecto (Cuarta Edición ed.). Bogotá, Colombia: Eco Ediciones.

Lesur, L. (2009). PUBLICIDAD Y PROPAGANDA (PRIMERA ed.). MÉXICO: TRILLAS S.A DE C.V.

License., G. G. (2017). Moodle. Obtenido de https:// docs.moodle.org/all/es/Acerca_de_Moodle

Lovelock, C., Reynoso, J., D’ Andrea, G., Huete, L., \& Wirtz, J. (2011). Administación de Servicio: Estrategias para la creación de valor en el nuevo paradigma de los negocios. México: Pearson Educación.

Malhotra, N. K. (2008). Investigación de Mercado (Quinta Edición ed.). (P. M. Guerrero Rosas, Ed.) Naucalpan de Juárez, Mexico: Pearson Education.

Méndez Álvarez, C. E. (2006). Metodología: Diseño y desarrollo del proceso de investigación. Limusa, Bogotá: Limusa S.a de C.V.

Muñoz, P. (2010). Consumidores, entretenimiento y comunicación de marketing en la era dl branded entertainment. España: Wolters Kluwer.

Olmos Gómez, M., \& Tomé Fernández, M. (2017). EVALUACIÓN DE INDICADORES DE CALIDAD DE LA SATISFACCIÓN DE LOS GRADOS DE. International Journal of Developmental and Educational Psychology.

Prieto Herrera, J. (2006). Merchandising. La Seducción en el Punto de Venta. Bogota: ecoe ediciones. 


\section{CITAR ESTE ARTICULO:}

Tomalá De la Cruz, M. A., Gallo Macías, G. G., Mosquera Viejó, J. L., \& Chancusig Chisag, J. C. (2020). Las plataformas virtuales para fomentar aprendizaje colaborativo en los estudiantes del bachillerato. RECIMUNDO, 4(4), 199-212. Recuperado a partir de http://recimundo.com/index.php/es/article/view/899

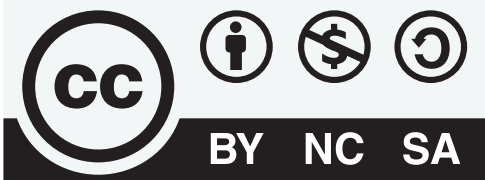

CREATIVE COMMONS RECONOCIMIENTO-NOCOMERCIAL-COMPARTIRIGUAL 4.0. 